\title{
From Gauge Anomalies to Gerbes and Gerbal Representations: Group Cocycles in Quantum Theory
}

\author{
J. Mickelsson
}

\begin{abstract}
In this paper I shall discuss the role of group cohomology in quantum mechanics and quantum field theory. First, I recall how cocycles of degree 1 and 2 appear naturally in the context of gauge anomalies. Then we investigate how group cohomology of degree 3 comes from a prolongation problem for group extensions and we discuss its role in quantum field theory. Finally, we discuss a generalization to representation theory where a representation is replaced by a 1-cocycle or its prolongation by a circle, and point out how this type of situations come up in the quantization of Yang-Mills theory.
\end{abstract}

\section{Introduction}

A projective bundle over a base $M$ is completely determined, up to equivalence, by the Dixmier-Douady class, which is an element of $H^{3}(M, \mathbf{Z})$. This is the origin of gerbes in quantum field theory: A standard example of this type of situation is the case when $M$ is the moduli space of gauge connections in a vector bundle over a compact spin manifold, [5]. Topologically a gerbe on a space $M$ is just an equivalence class of $P U(H)=U(H) / S^{1}$ bundles over $M$. Here $U(H)$ is the (contractible) unitary group in a complex Hilbert space $H$. In terms of Čech cohomology subordinate to a good cover $\left\{U_{\alpha}\right\}$ of $X$, the gerbe is given as a $\mathbf{C}^{\times}$-valued cocycle $\left\{f_{\alpha \beta \gamma}\right\}$,

$$
f_{\alpha \beta \gamma} f_{\alpha \beta \delta}^{-1} f_{\alpha \gamma \delta} f_{\beta \gamma \delta}^{-1}=1
$$

on intersections $U_{\alpha} \cap U_{\beta} \cap U_{\gamma} \cap U_{\delta}$. This cocycle arises from the lifting problem: A $P U(H)$ bundle is given in terms of transition functions $g_{\alpha \beta}$ with values in $P U(H)$. After lifting these to $U(H)$ one gets a family of functions $\hat{g}_{\alpha \beta}$ which satisfy the 1-cocycle condition up to a phase,

$$
\hat{g}_{\alpha \beta} \hat{g}_{\beta \gamma} \hat{g}_{\gamma \alpha}=f_{\alpha \beta \gamma} \mathbf{1} \text {. }
$$

The notion of gerbal representation was introduced in recent paper [7]. This is to be viewed as the next level after projective actions related to central extensions of groups and is given in terms of third group cohomology. One can view this setting as a categorification of the representation theory of central extensions of groups. We shall not discuss the problem in this generality since our categories are of special kind: A category of groups for us is just a principal bundle over a base $M$. Each fiber can be identified as a group $G$, but only after fixing a point in the fiber and calling the chosen point the unit element in $G$. Fixing a representation of $G$ defines a standard way a vector bundle over $M$. However, if the representation is only a projective representation we obtain in general only a projective vector bundle over $M$. We are now in the setting for gerbes and we have a characteristic class in $H^{3}(M, \mathbf{Z})$. But there is a role also for third group cohomology.

In fact, the appearance of third group cohomology in this context is not new and is related to group extensions as explained in [9]. In the simplest form, the problem is the following. Let $F$ be an extension of $G$ by the group $N$,

$$
1 \rightarrow N \rightarrow F \rightarrow G \rightarrow 1
$$

an exact sequence of groups. Suppose that $1 \rightarrow a \rightarrow$ $\hat{N} \rightarrow N \rightarrow 1$ is a central extension by the abelian group $a$. Then one can ask whether the extension $F$ of $G$ by $N$ can be prolonged to an extension of $G$ by the group $\hat{N}$. The obstruction to this is an element in the group cohomology $H^{3}(G, a)$ with coefficients in $a$. In the case of Lie groups, there is a corresponding Lie algebra cocycle representing a class in $H^{3}(\mathfrak{g}, \mathfrak{a})$, where $\mathfrak{a}$ is the Lie algebra of $a$. We shall demonstrate this in detail for an example arising from the quantization of gauge theory. It is closely related to the idea in [2], further elaborated in [3], which in turn was a response to a discussion in 1985 on breaking of the Jacobi identity for the field algebra in Yang-Mills theory [6].

The paper is organized as follows. In Section 2 we recall the basics about the role of group cohomology of degree 1 and 2 in quantum theory. In Section 3 we then explain how 3-cocycles come from a prolongation problem for group extensions. In Section 4 we take as an example the gauge group extensions arising from the gauge action on bundles of fermionic Fock spaces over background gauge fields and the corresponding Lie algebra cocycles. In Sections 5 and 6 we explain the use of 1-cocycles as generalized representations, with an example from quantum field theory.

\section{Cocycles of degree 1 and 2 in quantum theory}

In quantum mechanics a symmetry group $G$ (e.g., the group of Galilean symmetries) acts on Schrödinger wave functions as 


$$
(T(g) \psi)(x)=\omega(g ; x) \psi\left(g^{-1} x\right)
$$

where $\omega(g ; x)$, for $g \in G$, is a (matrix valued) phase factor. In order that the group multiplication rule is preserved it has to satisfy as consistency the 1-cocycle condition

$$
\omega\left(g_{1} ; x\right) \omega\left(g_{2} ; g_{1}^{-1} x\right) \omega\left(g_{1} g_{2} ; x\right)^{-1}=1 .
$$

It may happen that the cocycle condition does not hold, for example in the case of the Galilean transformations on wave functions of massive particles; in this case the left-hand-side defines a $S^{1}$ valued (it does not depend on the coordinate $x$ ) 2-cocycle. The representation of the Galilei group is now projective but it can still be viewed as a true representation for a central extension $\hat{G}$ of $G,[1]$.

1-cocycles appear also in the context of symmetry breaking in QFT. Classically, one might expect that the (exponentiated) quantum action is invariant under a group $G$ (group of gauge symmetries or group of diffeomorphisms of space-time),

$$
Z(A)=Z\left(A^{g}\right)
$$

where $A$ denotes a set of fields; in the case of a gauge action the right action is $A^{g}=g^{-1} A g+g^{-1} d g$. But in case of chiral anomaly, for example,

$$
Z\left(A^{g}\right)=\omega(g ; A) Z(A)
$$

where $\omega$ is a phase factor. Consistency requires again that $\omega$ is a 1-cocycle. However, unlike in the case of the Galilei group, the nontrivial 1-cocycle has serious physical consequences: It signals the breaking of gauge symmetry. Nontriviality means that there is no way to modify the quantum effective action by a multiplicative phase, $Z(A) \mapsto Z^{\prime}(A)=\eta(A) Z(A)$, such that the modified action $Z^{\prime}$ would be gauge invariant. This means that

$$
\omega(g ; A) \neq \eta\left(A^{g}\right) \eta(A)^{-1}
$$

for any phase function $\eta$. In case of an equality, we say that the 1-cocycle $\omega$ is a coboundary of the 0-cochain $\eta$.

Denote by $\mathcal{A}$ the space of all (smooth) fields $A$. If $G$ acts smoothly and freely on $\mathcal{A}$ then $X=\mathcal{A} / G$ is a manifold and the cocycle $\omega$ defines a complex line bundle $L$. Sections of $L$ are complex functions on $\mathcal{A}$ satisfying

$$
\psi\left(A^{g}\right)=\omega(g ; A) \psi(A) .
$$

The complex line bundle has Chern class $c \in H^{2}(X, \mathbf{Z})$ which is obtained by transgression from $\omega$. In the case of the chiral anomaly, the action $Z$ is thus a section of the complex line bundle $L$, which is called the Dirac determinant bundle. Indeed, the function $Z(A)$ can be viewed as a regularized determinant of the massless Weyl-Dirac operator $D_{A}^{+}$which is a linear map from the left-handed spinor fields to the right-handed sector. To define the determinant, one fixes a map $D_{A_{0}}^{-}$ from right-handed sector to the left-handed sector, by fixing a background potential $A_{0}$, and then one applies the zeta function regularization to the determinant of the operator $D_{A_{0}}^{-} D_{A}^{+}$.

In Hamiltonian quantization the breaking of (gauge, diffeomorphism) symmetry is best seen in the modified commutation relations in the Lie algebra $\mathfrak{g}$ of $G$,

$$
[X, Y] \mapsto[X, Y]+c(X, Y)
$$

where $c$ takes values in an abelian ideal $\mathfrak{a}$; in the simplest case $\mathfrak{a}=\mathbf{C}$ and $c$ satisfies the Lie algebra 2-cocycle condition

$$
c(X,[Y, Z])+c(Y,[Z, X])+c(Z,[X, Y])=0
$$

A famous example is given by the central extension of the loop algebra $L \mathfrak{g}$ of smooth functions on the unit circle with values in a semisimple Lie algebra $\mathfrak{g}$,

$$
c(X, Y)=\frac{k}{2 \pi i} \int_{S^{1}}\left\langle X(\phi), Y^{\prime}(\phi)\right\rangle \mathrm{d} \phi
$$

where $k$ is a constant ("level" of a representation), which is equal to a nonnegative integer in a positive energy representation when the invariant bilinear form $\langle\cdot, \cdot\rangle$ on $\mathfrak{g}$ is properly normalized.

Given a (central) extension of a Lie algebra one expects that there is a central extension of the corresponding group. In case of $L \mathfrak{g}$ the group is the loop group $L G$ of maps from $S^{1}$ to a (compact) group $G$. A central extension of $L G$ would then be given by a circle valued function $\Omega: L G \times L G \rightarrow S^{1}$ with group 2-cocycle property

$$
\Omega\left(g_{1}, g_{2}\right) \Omega\left(g_{1} g_{2}, g_{3}\right)=\Omega\left(g_{1}, g_{2} g_{3}\right) \Omega\left(g_{2}, g_{3}\right) .
$$

However, in case of $L G$ there is a topological obstruction, $\Omega$ is defined only in an open neighborhood of the unit element. The obstruction is given by an element in $H^{2}(L G, \mathbf{Z})$ whose de Rham representative is a left invariant 2 -form fixed by the Lie algebra 2-cocycle $c$. In case of a compact simple simply connected Lie group the cohomology $H^{2}(L G, \mathbf{Z})$ is equal to $H^{3}(G, \mathbf{Z})$ is equal to $\mathbf{Z}$. The Lie algebra cocycle for $L \mathfrak{g}$ can be viewed as a left invariant 2 -form on the group $L G$. For a correct choice of normalization of the bilinear form $\langle, \cdot, \cdot\rangle$ on $\mathfrak{g}$ the generator in $H^{2}(L G, \mathbf{Z})$ corresponds to the basic extension with level $k=1$.

\section{3-cocycles}

Group and Lie algebra cohomology with coefficients in an abelian group (Lie algebra) is defined in any degree. So what about degree 3? And the relation to de Rham cohomology in dimension 3 ?

First, let us recall the basic definitions. Assume that a group $G$ acts as automorphisms of an abelian group $A$. A map $f: G \times G \times \ldots G: \rightarrow A$ ( $n$ arguments) 
is a n-cocycle if $\delta f=0$ where the coboundary operator $\delta$ is defined by

$$
\begin{aligned}
& (\delta f)\left(g_{1}, g_{2}, \ldots, g_{n+1}\right)= \\
& \sum_{i=1}^{i=n}(-1)^{i} f\left(g_{1}, \ldots g_{i} g_{i+1}, \ldots, g_{n+1}\right)+ \\
& (-1)^{n+1} f\left(g_{1}, \ldots, g_{n}\right)+g_{1} \cdot f\left(g_{2}, \ldots, g_{n+1}\right) .
\end{aligned}
$$

Cocycles of type $\delta f$ are exact cocycles. The group cohomology in degree $n$ is then defined as the abelian group $H^{n}(G ; A)$ of n-cocycles modulo exact cocycles.

In case of a Lie algebra $\mathfrak{g}$ the cochains are alternating multilinear maps $f: \mathfrak{g} \times \ldots \times \mathfrak{g} \rightarrow \mathfrak{a}$. The cocycles are elements in the kernel of the Lie algebra coboundary operator, which is now defined as

$$
\begin{aligned}
& (\delta f)\left(x_{1}, \ldots, x_{n+1}\right)= \\
& \sum_{i<j}(-1)^{i+j+1} f\left(\left[x_{i}, x_{j}\right], x_{1}, \ldots \hat{x}_{i} \ldots \hat{x}_{j} \ldots x_{n+1}\right)+ \\
& \sum_{i=1}^{i=n+1}(-1)^{i} x_{i} \cdot f\left(x_{1}, \ldots \hat{x}_{i} \ldots x_{n+1}\right),
\end{aligned}
$$

where the argument under the hat is deleted. Here the Lie algebra $\mathfrak{g}$ acts as endomorphisms of the abelian Lie algebra $\mathfrak{a}$. The Lie algebra cohomology $H^{n}(\mathfrak{g} ; \mathfrak{a})$ is now the abelian group of $n$-cocycles modulo exact $n$-cocycles.

Let $\mathcal{B}$ be an associative algebra and $G$ a group. Assume that we have a group homomorphism $s: G \rightarrow$ $\operatorname{Out}(\mathcal{B})$ where $\operatorname{Out}(\mathcal{B})$ is the group of outer automorphisms of $\mathcal{B}$, that is, $\operatorname{Out}(\mathcal{B})=\operatorname{Aut}(\mathcal{B}) / \operatorname{In}(\mathcal{B})$, all automorphisms modulo the normal subgroup of inner automorphisms.

If one chooses any lift $\tilde{s}: G \rightarrow \operatorname{Aut}(\mathcal{B})$ then we can write

$$
\tilde{s}(g) \tilde{s}\left(g^{\prime}\right)=\sigma\left(g, g^{\prime}\right) \cdot \tilde{s}\left(g g^{\prime}\right)
$$

for some $\sigma\left(g, g^{\prime}\right) \in \operatorname{In}(\mathcal{B})$. From the definition follows immediately the cocycle property

$$
\sigma\left(g, g^{\prime}\right) \sigma\left(g g^{\prime}, g^{\prime \prime}\right)=\left[\tilde{s}(g) \sigma\left(g^{\prime}, g^{\prime \prime}\right) \tilde{s}(g)^{-1}\right] \sigma\left(g, g^{\prime} g^{\prime \prime}\right)
$$

Let next $H$ be any central extension of $\operatorname{In}(\mathcal{B})$ by an abelian group $a$. That is, we have an exact sequence of groups,

$$
1 \rightarrow a \rightarrow H \rightarrow \operatorname{In}(\mathcal{B}) \rightarrow 1
$$

Let $\hat{\sigma}$ be a lift of the map $\sigma: G \times G \rightarrow \operatorname{In}(\mathcal{B})$ to a map $\hat{\sigma}: G \times G \rightarrow H$ (by a choice of a section $\operatorname{In}(\mathcal{B}) \rightarrow H$ ). We have then

$$
\begin{array}{r}
\hat{\sigma}\left(g, g^{\prime}\right) \hat{\sigma}\left(g g^{\prime}, g^{\prime \prime}\right)=\left[\tilde{s}(g) \hat{\sigma}\left(g^{\prime}, g^{\prime \prime}\right) \tilde{s}(g)^{-1}\right] \\
\times \hat{\sigma}\left(g, g^{\prime} g^{\prime \prime}\right) \cdot \alpha\left(g, g^{\prime}, g^{\prime \prime}\right) \text { for all } g, g^{\prime}, g^{\prime \prime} \in G
\end{array}
$$

where $\alpha: G \times G \times G \rightarrow a$.

Here the action of the outer automorphism $s(g)$ on $\hat{\sigma}(*)$ is defined by $\tilde{s}(g) \hat{\sigma}(*) \tilde{s}(g)^{-1}=$ the lift of $\tilde{s}(g) \sigma(*) \tilde{s}(g)^{-1} \in \operatorname{In}(\mathcal{B})$ to an element in $H$. One can show that $\alpha$ is a 3-cocycle, [9],

$$
\begin{array}{r}
\alpha\left(g_{2}, g_{3}, g_{4}\right) \alpha\left(g_{1} g_{2}, g_{3}, g_{4}\right)^{-1} \alpha\left(g_{1}, g_{2} g_{3}, g_{4}\right) \\
\times \alpha\left(g_{1}, g_{2}, g_{3} g_{4}\right)^{-1} \alpha\left(g_{1}, g_{2}, g_{3}\right)=1
\end{array}
$$

where we have used the multiplicative notation for the group product in $a$ (instead of the additive notation in the definition of the coboundary operator). The group $G$ acts trivially on $a$.

Remark If we work in the category of topological groups (or Lie groups) the lifts above are in general discontinuous; normally, we can require continuity (or smoothness) only in an open neighborhood of the unit element.

\section{A QFT example}

Next we construct an example from quantum field theory. Let $G$ be a compact simply connected Lie group and $P$ the space of smooth paths $f:[0,1] \rightarrow G$ with initial point $f(0)=e$, the neutral element, and quasiperiodicity condition $f^{-1} d f$ a smooth function.

$P$ is a group under point-wise multiplication but it is also a principal $\Omega G$ bundle over $G$. Here $\Omega G \subset P$ is the based loop group with $f(0)=f(1)=e$ and $\pi: P \rightarrow G$ is the projection to the end point $f(1)$. Fix an unitary representation $\rho$ of $G$ in $\mathbf{C}^{N}$ and denote $\mathcal{H}=L^{2}\left(S^{1}, \mathbf{C}^{N}\right)$.

For each polarization $\mathcal{H}=\mathcal{H}_{-} \oplus \mathcal{H}_{+}$we have a vacuum representation of the canonical anticommutation relations algebra $(\mathrm{CAR}) \mathcal{B}(\mathcal{H})$ in a Hilbert space $\mathcal{F}\left(\mathcal{H}_{+}\right)$. Denote by $\mathcal{C}$ the category of these representations. Denote by $a(v), a^{*}(v)$ the generators of $\mathcal{B}(\mathcal{H})$ corresponding to a vector $v \in \mathcal{H}$,

$$
a^{*}(u) a(v)+a(v) a^{*}(u)=2\langle v, u\rangle \cdot \mathbf{1}
$$

and all the other anticommutators equal to zero.

Any element $f \in P$ defines a unique automorphism of $\mathcal{B}(\mathcal{H})$ with $\phi_{f}\left(a^{*}(v)\right)=a^{*}(f \cdot v)$, where $f \cdot v$ is the function on the circle defined by $\rho(f(x)) v(x)$. These automorphisms are in general not inner except when $f$ is periodic.

We have now a map $s: G \rightarrow \operatorname{Aut}(\mathcal{B}) / \operatorname{In}(\mathcal{B})$ given by $g \mapsto F(g)$ where $F(g)$ is an arbitrary smooth quasiperiodic function on $[0,1]$ such that $F(g)(1)=g$.

Any two such functions $F(g), F^{\prime}(g)$ differ by an element $\sigma$ of $\Omega G, F(g)(x)=F^{\prime}(g)(x) \sigma(x)$. Now $\sigma$ is an inner automorphism through a projective representation of the loop group $\Omega G$ in $\mathcal{F}\left(\mathcal{H}_{+}\right)$.

In an open neighborhood $U$ of the neutral element $e$ in $G$ we can fix in a smooth way for any $g \in U$ a path $F(g)$ with $F(g)(0)=e$ and $F(g)(1)=g$.

Of course, for a connected group $G$ we can make this choice globally on $G$ but then the dependence of the path $F(g)$ would not be a continuous function of the end point. For a pair $g_{1}, g_{2} \in G$ we have 


$$
\sigma\left(g_{1}, g_{2}\right) F\left(g_{1} g_{2}\right)=F\left(g_{1}\right) F\left(g_{2}\right)
$$

with $\sigma\left(g_{1}, g_{1}\right) \in \Omega G$.

For a triple of elements $g_{1}, g_{2}, g_{3}$ we have now

$$
\begin{aligned}
F\left(g_{1}\right) F\left(g_{2}\right) F\left(g_{3}\right) & =\sigma\left(g_{1}, g_{2}\right) F\left(g_{1} g_{2}\right) F\left(g_{3}\right) \\
& =\sigma\left(g_{1}, g_{2}\right) \sigma\left(g_{1} g_{2}, g_{3}\right) F\left(g_{1} g_{2} g_{3}\right) .
\end{aligned}
$$

In the same way,

$$
\begin{aligned}
& F\left(g_{1}\right) F\left(g_{2}\right) F\left(g_{3}\right)=F\left(g_{1}\right) \sigma\left(g_{2}, g_{3}\right) F\left(g_{2} g_{3}\right)= \\
& {\left[g_{1} \sigma\left(g_{2}, g_{3}\right) g_{1}^{-1}\right] F\left(g_{1}\right) F\left(g_{2} g_{3}\right)=} \\
& {\left[g_{1} \sigma\left(g_{2}, g_{3}\right) g_{1}^{-1}\right] \sigma\left(g_{1}, g_{2} g_{3}\right) F\left(g_{1} g_{2} g_{3}\right)}
\end{aligned}
$$

which proves the 2-cocycle relation for $\sigma$.

Lifting the loop group elements $\sigma$ to inner automorphisms $\hat{\sigma}$ through a projective representation of $\Omega G$ we can write

$$
\begin{aligned}
& \hat{\sigma}\left(g_{1}, g_{2}\right) \hat{\sigma}\left(g_{1} g_{2}, g_{3}\right)= \\
& \operatorname{Aut}\left(g_{1}\right)\left[\hat{\sigma}\left(g_{2}, g_{3}\right)\right] \hat{\sigma}\left(g_{1}, g_{2} g_{3}\right) \alpha\left(g_{1}, g_{2}, g_{3}\right),
\end{aligned}
$$

where $\alpha: G \times G \times G \rightarrow S^{1}$ is some phase function arising from the fact that the projective lift is not necessarily a group homomorphism.

Since (in the case of a Lie group) the function $F(\cdot)$ is smooth only in a neighborhood of the neutral element, the same is true also for $\sigma$ and finally for the 3-cocycle $\alpha$.

An equivalent point of view to the construction of the 3 -cocycle $\alpha$ is this: We are trying to construct a central extension $\hat{P}$ of the group $P$ of paths in $G$ (with initial point $e \in G$ ) as an extension of the central extension over the subgroup $\Omega G$. The failure of this central extension is measured by the cocycle $\alpha$, as an obstruction to associativity of $\hat{P}$.

On the Lie algebra level, we have a corresponding cocycle $c_{3}=d \alpha$ which is easily computed. The cocycle $c$ of $\Omega \mathfrak{g}$ extends to the path Lie algebra $P \mathfrak{g}$ as

$$
c(X, Y)=\frac{k}{4 \pi i} \int_{[0,2 \pi]}(\langle X, \mathrm{~d} Y\rangle-\langle Y, \mathrm{~d} X\rangle) .
$$

This is an antisymmetric bilinear form on $P \mathfrak{g}$ but it fails to be a Lie algebra 2-cocycle. The coboundary is given by

$$
\begin{aligned}
& (\delta c)(X, Y, Z)= \\
& c(X,[Y, Z])+c(Y,[Z, X])+c(Z,[X, Y])= \\
& -\left.\frac{k}{4 \pi i}\langle X,[Y, Z]\rangle\right|_{2 \pi}=d \alpha(X, Y, Z) .
\end{aligned}
$$

Thus $\delta c$ reduces to a 3 -cocycle of the Lie algebra $\mathfrak{g}$ of $G$ on the boundary $x=2 \pi$. Assuming that the bilinear form is normalized as $\langle X, Y\rangle=\operatorname{tr} X Y$, trace in the defining representation of $G$, then the above 3 -cocycle defines by left translations on $G$ the left-invariant de Rham form $-\frac{1}{12 \pi i} \operatorname{tr}\left(g^{-1} d g\right)^{3}$; this is normalized as
$2 \pi i$ times an integral 3-form on $G$ and is the generator of $H^{3}(G, \mathbf{Z})$ for $G=S U(n)$.

\section{Cocycles and associated vector bundles}

Let us recall the standard construction of a vector bundle associated to a principal $G$ bundle $\pi: P \rightarrow M$ over a base $M$. Fix a representation $\rho: G \rightarrow \operatorname{Aut}(V)$, in a vector space $V$. Define the total space of a vector bundle as

$$
E=P \times_{\rho} V,
$$

with the equivalence

$$
(p, v) \equiv\left(p g, \rho(g)^{-1} v\right) .
$$

The projection onto the base $M$ is $(p, v) \mapsto \pi(p)$. Consider the following generalization of this construction: Fix a 1-cocycle $\omega: P \times G \rightarrow \operatorname{Aut}(V)$ and set

$$
E=P \times{ }_{\omega} V, \text { with }\left(p g, \omega(p ; g)^{-1} v\right) \equiv(p, v) .
$$

The transitivity of the relation is given by the cocycle condition

$$
\omega\left(p ; g g^{\prime}\right)=\omega(p ; g) \omega\left(p g ; g^{\prime}\right) .
$$

An example of this construction was already given in the construction of the determinant bundle over the gauge orbit space $\mathcal{A} / \mathcal{G}$.

Let $G$ be a topological group and $f: G \rightarrow H$ a homotopy to another topological group $H$. Morally, representation theory of $H$ should encode information about representations of $G$. However, it can happen that $H$ has a good representation theory but $G$ lacks unitary faithful Hilbert space representations. But we can define a cocycle

$$
\omega(b ; g)=f(b)^{-1} f(b g)
$$

with values in $H$. Selecting a representation $\rho$ of $H$ in $V$ we obtain a 1 -cocycle $\rho(\omega(b ; g))$ for the right action of $G$ on itself, with values in $\operatorname{Aut}(V)$. We can view this as a representation of $G$ in a group of matrices with entries in the algebra of complex functions on $G$ (but with an action of $G$ on functions through right translation).

Example 1 The loop group $L G$ of smooth maps $f: S^{1} \rightarrow G, G$ a compact Lie group, has a beautiful theory of projective highest weight representations, $[11,10]$. These are representations of a central extension $\widehat{L G}$. On the Lie algebra level, the central extension is given as in Sect. 3.

One can show that $L G$ is homotopy equivalent to the Banach-Lie group $L^{c} G$ of continuous loops, [4]. However, no representations of $L^{c} G$ analogous to the highest weight representations are known. Instead, we can use the cocycle

$$
\omega: L^{c} G \times L^{c} G \rightarrow L G
$$


to tranfer representations of $L G$ to Hilbert space operator cocycles on $L^{c} G,[8]$.

Example 2 Let $\mathcal{H}=\mathcal{H}_{-} \oplus \mathcal{H}_{+}$be a polarized Hilbert space and $U_{p}$ the group of unitaries in $\mathcal{H}$ such that the off-diagonal blocks with respect to the polarization are in the Schatten ideal $L_{p}$ of bounded operators $A$ with $\operatorname{tr}|A|^{p}<\infty$. The case $p=2$ is important since the highest weight representations of $\widehat{L G}$ can be constructed from representations of a central extension $\hat{U}_{2},[11]$. The Lie algebra central extension is defined by the 2-cocycle

$$
c(X, Y)=\frac{1}{2} \operatorname{tr}_{c} X[\epsilon, Y]
$$

where $\epsilon$ is the grading operator in $\mathcal{H}$ and the condition $\operatorname{trace} \operatorname{tr}_{c}$ is defined as $\operatorname{tr}_{c} X=\frac{1}{2} \operatorname{trtr}(X+\epsilon X \epsilon)$. The groups $U_{p}$ are important because one has an embedding $\operatorname{Map}(M, G) \subset U_{p}$ when $M$ is a compact spin manifold and $G$ compact Lie group, for $p>\operatorname{dim} M$, [15]. According to Richard Palais, $U_{p}$ is homotopy equivalent to $U_{2}$ for all $p \geq 1,[16]$, so we can define generalized representations of $\operatorname{Map}(M, G)$ from this equivalence and the embebding to $U_{p}$.

\section{Application to gauge theory}

Let $D_{A}$ Dirac hamiltonian on an odd dimensional compact spin manifold coupled to a gauge potential $A$. The quantization $\hat{D}_{A}$ of $D_{A}$ acts in a fermionic Fock space. For different potentials the representations of the fermion algebra are inequivalent [17]. In scattering problems one would like to realize the operators $\hat{D}_{A}$ in a single Fock space $\mathcal{F}$, the Fock space of free fermions, $A=0$. This can be achieved by choosing for each $A$ a unitary operator $T_{A}$ which reduces the offdiagonal blocks of $D_{A}$ to Hilbert-Schmidt operators, for the 'free' polarization $\epsilon=D_{0} /\left|D_{0}\right|$. Then each $D_{A}^{\prime}=T_{A}^{-1} D_{A} T_{A}$ can be quantized in the free Fock space, $[12],[13]$.

This has a consequence for the implementation of the gauge action $A \mapsto A^{g}=g^{-1} A g+g^{-1} d g$ in the Fock space. In the 1-particle space the action of $g$ is replaced by $\omega(A ; g)=T_{A}^{-1} g T_{A^{g}}$ with

$$
\omega\left(A ; g g^{\prime}\right)=\omega(A ; g) \omega\left(A^{g} ; g^{\prime}\right) .
$$

Now the Shale-Stinespring condition $[\epsilon, \omega(A ; g)] \in$ $L_{2}$ is satisfied and we can quantize in $\mathcal{F}$,

$$
\omega(A ; g) \mapsto \hat{\omega}\left(A ; g^{\prime}\right)
$$

For the Lie algebra of the gauge group we have the Lie algebra cocycle

$$
d \omega(A ; X)=T_{A}^{-1} X T_{A}+T_{A}^{-1} \mathcal{L}_{X} T_{A}
$$

with quantization $\widehat{d \omega}(A ; X)$.

Let $X$ be an element in the Lie algebra $\operatorname{Map}(M, \mathfrak{g})$ of the gauge group $\mathcal{G}=\operatorname{Map}(M, G)$. Its quantization is then

$$
G_{X}=\mathcal{L}_{X}+\widehat{d \omega}(A ; X)
$$

where $\mathcal{L}_{X}$ is the Lie derivative in the direction of $X$, corresponding to the infinitesimal gauge action $A \mapsto[A, X]+d X$. The commutation relations are now modified by a cocycle $c$,

$$
\left[G_{X}, G_{Y}\right]=G_{[X, Y]}+c(A ; X, Y)
$$

$\mathcal{L}_{X} c(A ;[Y, Z])+c(A ;[X,[Y, Z]])+$ cyclic combin. $=0$.

The Lie derivative $\mathcal{L}_{X}$ is needed since the Fock spaces $\mathcal{F}$ depend on the external gauge field $A$ : although as Hilbert spaces $\mathcal{F}_{A}$ are all the same, the gauge action explicitly depends on $A$. The 2-cocycle property quarantees the Jacobi identity for the extension

$$
\operatorname{Lie}(\hat{\mathcal{G}})=\operatorname{Map}(M, \mathfrak{g}) \oplus \operatorname{Map}(\mathcal{A}, \mathbf{C}) .
$$

In the case when $M=S^{1}$ one can take $T_{A}=1$ and we obtain the standard central extension of the loop algebra $\operatorname{Map}\left(S^{1}, \mathfrak{g}\right)$. In the case when $\operatorname{dim} M=3$ one can show that the 2-cocycle $c$ is equivalent to the local form

$$
c \equiv \text { const. } \int_{M} \operatorname{tr} A[\mathrm{~d} X, \mathrm{~d} Y]
$$

where the trace under the integral sign is computed in a finite-dimensional representation of $\mathfrak{g}$. This representation is the same defined by the $G$-action on fermions in the 1-particle space. Actually, the coefficient in the front of the integral is nonzero only for chiral fermions (the Schwinger terms from left and right chiral sectors cancel).

\section{References}

[1] Bargmann, V.: On unitary ray representations of continuous groups. Ann. of Math. (2) 59, (1954), $1-46$.

[2] Carey, A. L.: The origin of three-cocycles in quantum field theory. Phys. Lett. B 194, (1987), 267-270.

[3] Carey, A. L., Grundling, H., Raeburn, I., Sutherland, C.: Group actions on $C^{*}$-algebras, 3cocycles and quantum field theory. Commun. Math. Phys. 168, (1995), 389-416.

[4] Carey, A. L., Crowley, D., Murray, M. K.: Principal bundles and the Dixmier Douady class. Comm. Math. Phys. 193, (1998), no. 1, 171-196.

[5] Carey, A. L., Mickelsson, J., Murray, M. K.: Bundle gerbes applied to quantum field theory. Rev. Math. Phys. 12, (2000), no. 1, 65-90. 
[6] Grossman, B.: The meaning of the third cocycle in the group cohomology of nonabelian gauge theories. Phys. Lett. B 160, (1985), 94-100. Jackiw, R.: Three-cocycle in mathematics and physics. Phys. Rev. Lett. 54, (1985), 159-162. Jo, S. G.: Commutator of gauge generators in nonabelian chiral theory. Nuclear Phys. B 259, (1985), 616-636.

[7] Frenkel, E., Xinwen Zhu: Gerbal representations of double loop groups. arXiv.math/0810.1487

[8] Hekmati, P., Mickelsson, J.: Fractional loop group and twisted K-theory. arXiv:0801.2522.

[9] Mac Lane, Saunders: Homology. Die Grundlehren der Mathematischen Wissenschaften, Band 114 Springer Verlag (1963).

[10] Kac, Victor: Infinite-dimensional Lie algebras. Third edition. Cambridge University Press, Cambridge, (1990).

[11] Pressley, A., Segal, G.: Loop Groups. Oxford Mathematical Monographs. The Clarendon Press, Oxford University Press, New York, (1986).

[12] Mickelsson, J.: Wodzicki residue and anomalies of current algebras. Integrable models and strings (Espoo, 1993), 123-135, Lecture Notes in Phys., 436, Springer, Berlin, (1994).
[13] Langmann, E., Mickelsson, J.: Scattering matrix in external field problems. J. Math. Phys. 37, (1996), no. 8, 3933-3953.

[14] Mickelsson, J.: From gauge anomalies to gerbes and gerbal actions. arXiv:0812.1640. To be publ. in the proceedings of "Motives, Quantum Field Theory, and Pseudodifferential Operators", Boston University, June 2-13, 2008.

[15] Mickelsson, J., Rajeev, S. G.: Current algebras in $d+1$-dimensions and determinant bundles over infinite-dimensional Grassmannians. Comm. Math. Phys. 116, (1988), no. 3, 365-400.

[16] Palais, R.: On the homotopy type of certain groups of operators. Topology 3, (1965), 271-279.

[17] Shale, David, Stinespring, W. F.: Spinor representations of infinite orthogonal groups. J. Math. Mech. 14, (1965), 315-322.

Jouko Mickelsson

Department of Mathematics and Statistics

University of Helsinki

Department of Theoretical Physics

Royal Institute of Technology, Stockholm 